Sobre Kant 



\title{
Problemas de la recepción de la tercera Crítica de Kant
}

\author{
Evodio Escalante
}

$\mathbf{L}$

a historia de la filosofía es también la historia de sus equívocos, y esto no constituye de modo obligado una prueba en contra de la interpretación filosófica de los textos. La fama, y todavía mejor, la trascendencia de un autor y de su obra, y con esto último aludo a la efectividad de su pensamiento lo mismo que a la cauda de sus repercusiones y sus inervaciones en el tiempo, está acompañada muy a menudo de lo que podríamos llamar un "desvarío armónico". La nota fundamental y con ella la línea melódica principal queda así oscurecida y lo que alcanza el primer plano es el acorde más o menos parásito del que se había servido para destacarse. A veces los armónicos se desligan de la nota con la que hacían sistema e ingresan en el vértigo de una atonalidad escalofriante, en el que la melodía antes dueña y señora se convierte en una humilde criada que se ocupa de las tareas más bajas. No hablemos de un cáncer de la interpretación, ni de una dislocación hermenéutica, sino del necesario y hasta indispensable tránsito de los textos, que se traducen de un pensador a otro sin tener que aflojar las fronteras de la lengua ni de la geografía. Hegel lector de Kant no me deja mentir. El armónico de la "subjetivización kantiana", y no sólo la que concierne a la estética, se convierte a partir de la intencionada lectura de Hegel en la nota sintética de un sistema que como quiera que se lo vea apunta siempre en direcciones más complejas. ${ }^{1}$ En lugar de la tónica, la dominante; en lugar de la dominante, la

${ }^{1}$ El dictum hegeliano de la subjetivización se adivina ya en una de sus obras tempranas, Fe y saber. O la filosofía de la reflexión de la subjetividad en la totalidad de sus formas como filosofía de Kant, Jacobi y Fichte. Trad. de Vicente Serrano. Madrid, Biblioteca Nueva, 2000, y lo confirma sin el menor reparo crítico Hans-Georg Gadamer en su obra Verdad y método. Trad. de Ana Agud Aparicio y Rafael de Agapito. Salamanca, Sígueme, 2001, cuando da por título de uno de sus capítulos "La subjetivización de la estética por la crítica kantiana". 
subdominante, siempre que no se caiga en la pura distorsión armónica o en el delirio de la atonalidad.

La trascendencia de la Crítica del juicio de Kant está hecha de aciertos pero también de felices malentendidos que no cesan de prolongarse hasta nuestros días. En su libro sobre Nietzsche, Martin Heidegger sostiene un poco al pasar: "Schopenhauer desempeña el papel principal en el surgimiento y preparación de la mala comprensión de la estética kantiana a la que también Nietzsche sucumbe y que aún hoy está en boga. Puede decirse que la Crítica del juicio de Kant, la obra en la que está expuesta su estética, sólo ha tenido efecto hasta ahora por obra de malentendidos, proceso corriente en la historia de la filosofía". ${ }^{2}$

Resulta paradójico observar que incluso Heidegger parece incurrir aquí en un primer malentendido, muy generalizado, y no sólo entre nosotros: el de considerar que Kant escribió una estética, el de estimar que la Crítica del juicio es una obra de estética, al menos en su primera parte, la cual, no se sabe bien por qué, habría sido publicada con otro nombre, con un título que quizás no le correspondería del todo. El eminente José Gaos aborda el asunto como si se tratara de una obviedad: "Mas basta hojear el índice de la Crítica del juicio, sin necesidad ni de hojear la obra misma, para ver que en ella se trata de la estética y de la concepción teleológica del organismo vegetal y animal..."3 Lo que yo veo delinearse aquí es la falacia del género. Se supone que a partir de Baumgarten existiría una disciplina a la que de cierto modo los filósofos tendrían que rendir tributo. La compulsión del género habría llevado a Kant a escribir, así sea de modo "accidental", como llega a sugerir de modo atrevido Gaos, una estética. Se olvida que ya desde la redacción de la Crítica de la razón pura, Kant había expresado una animadversión a propósito de este término y lo que él implica: una disciplina rigurosa y científica acerca del arte y sus principios rectores. ${ }^{4}$ Se olvida -o bien no se ha reparado

${ }^{2}$ Martin Heidegger, Nietzsche. Trad. de José Luis Vermal. Barcelona, Destino, 2000 , t. I, p. 109.

${ }^{3} \mathrm{Al}$ final de su texto, Gaos matiza de un modo que no deja de ser problemático, y que suscita nuevas interrogaciones: "El sistema de las tres Críticas kantianas es esencialmente un sistema de encierro de la razón pura en los fenómenos, para dejar a la razón práctica abierta a los nóumenos, completado accidentalmente con una estética..." (vid. José Gaos, "Las Críticas de Kant", en De Descartes a Marx. Estudios y notas de historia de la filosofía. Obras completas.. México, UNAM, 1977, t. IV, pp. 323 y 337 . El subrayado es mío).

${ }^{4}$ Remito a la nota con la que se abre el capítulo dedicado a la "Estética trascendental" en la Crítica de la razón pura. Con sorna no disimulada observa Kant: "Los alemanes son los únicos que emplean hoy la palabra 'estética' para designar lo que otros denominan crítica del gusto. Tal empleo se basa en la equivocada esperanza 
siquiera en ello- que de manera explícita el propio Kant advierte en un pasaje de la Crítica del juicio acerca de la imposibilidad radical de una ciencia de lo bello, con lo que de modo automático quedaría descartada la posibilidad de concebir una disciplina estética. Sostiene Kant, a la letra: "No hay ni una ciencia de lo bello, sino una crítica, ni una ciencia bella, sino sólo arte bella, pues en lo que se refiere a la primera, debería determinarse científicamente, es decir, con bases de demostración, si hay que tener algo por bello o no; el juicio sobre la belleza, si perteneciese a la ciencia, no sería juicio alguno de gusto". 5

Cuando Kant decide escribir la Crítica del juicio, no lo hace para cubrir un supuesto hueco de género en su pensamiento, lo que estoy seguro le tenía sin cuidado, sino porque ha descubierto, contra sus previsiones anteriores expresadas en la nota citada de la primera de las Críticas, a la que hay que regresar cada vez que sea necesario, que podía colocar un principio a priori en la base del juicio reflexionante, el cual se dividiría a su vez en juicio estético y teleológico. Al descubrir esta base apriorística, fundamento de su posición radical en filosofía, Kant reanuda una investigación minuciosa cuyo tema es ni más ni menos que el juicio que él llama reflexionante. El libro de Kant no se nos propone, pues, como una estética, ni siquiera como un esbozo o boceto de la misma. De hecho es a la vez mucho menos y mucho más que una estética. Es menos que una estética porque se constriñe a ser lo que el título anticipa, una crítica del juicio, en este caso el juicio estético; es más que una estética, porque al ocuparse del juicio teleológico atribuye así sea sólo con propósitos heurísticos una finalidad a la naturaleza orgánica e inorgánica. Si con esto último desborda los límites de una disciplina, con lo primero se nos aparece Kant como un pensador minimalista, que ha asumido el rigor de la autolimitación, empeñado en explorar tan sólo un pequeño lote baldío, el que él ha descubierto, sin salir jamás de éste. No cree que pueda haber una ciencia de lo bello como tal, como él mismo declara, aunque sí una crítica aplicada a un determinado tipo de juicio. Si desplaza la posibilidad de una estética en forma, es porque se ocupará (nada más, pero nada menos) de explorar cómo funciona el juicio estético. El título de la obra obliga a enten-

concebida por el destacado crítico Baumgarten". Ahí mismo aconseja o suprimir esa denominación o bien reservarla (como él mismo hace en este libro) "para la doctrina que constituye una verdadera ciencia" -se refiere, por supuesto, a la ciencia de la percepción, a la de la intuición sensible, nada que ver con lo que entendemos estos días por estética. Vid. Immanuel Kant, Crítica de la razón pura. Trad. de Pedro Ribas. Madrid, Alfaguara, 2000, pp. 66-67

${ }^{5}$ I. Kant, Crítica del juicio. Trad. de Manuel García Morente. México, Porrúa, 1991 (Sepan Cuantos, 246), cap. 44, p. 277. Los subrayados son míos. 
derlo en su sentido a la vez estricto y literal, aunque escuchando las resonancias inevitables que esta literalidad conlleva. Es una Crítica del juicio, o bien como proponen algunos, no sin razón, una Crítica de la facultad de juzgar. Tan cercano a menudo a la terminología jurídica, a la que nunca despoja de su valor técnico, no podría descartarse sin embargo que este título kantiano aluda de alguna forma a lo que sería una jurisprudencia en los asuntos de lo bello y lo sublime, como lo sugiere su idea de un "sentido común estético", que estaría de alguna manera en proceso de formación.

Por otra parte, en el prólogo de este libro el autor advierte, para que nadie pueda llamarse a engaño: "La investigación de la facultad del gusto como Juicio estético se expone aquí, no para la formación y cultivo del gusto [...], sino con una intención trascendental; me complazco en pensar, por lo tanto, que en lo que toca a la imperfección de aquel primer asunto, será juzgada con indulgencia; pero en lo que toca al segundo, debe estar prevenida para el examen más severo". ${ }^{6}$ La intención trascendental consistiría, si entiendo bien, en aportar la fundamentación apriorística del juicio reflexionante en sus dos modalidades. Esta intención está justificada con creces. Permanece fuera de sus propósitos, en cambio, como queda patente, y por eso solicita ser juzgado con indulgencia, contribuir con esta Crítica del juicio a "la formación y cultivo del gusto”. Además de una obvia razón sistemática, no es difícil conjeturar que esto se debe a que Kant era muy consciente de sus limitaciones personales en este campo de la cultura. No lo digo para denostar a Kant, sino para admirarme todavía más de la extraordinaria vigencia de su obra. La cultura artística de Kant era no sólo pobre sino provinciana, y creo que estaba bastante por debajo del nivel medio que se le pediría a un hombre de ciencia de su época. A Kant le parece más interesante y mucho más variado, por ejemplo, el trinar de los pájaros que las modulaciones de la voz humana. No le levanto falsos al filósofo de Königsberg, por eso lo cito: "El canto mismo de los pájaros, que no podemos reducir a reglas musicales, parece encerrar más libertad y, por tanto, más alimento para el gusto que el canto humano mismo dirigido según todas las reglas musicales, porque este último más bien hastía cuando se repite muchas veces y durante largo tiempo". ${ }^{7}$

En la época del apogeo de Haydn y de la celebridad de Mozart, Kant sostiene que hay en la música "una cierta falta de urbanidad". Entre las bellas artes, la música ocuparía un lugar inferior según el grado de cultura que despierta

${ }^{6}$ Ibid., p. 187. El subrayado es mío.

${ }^{7}$ Ibid., cap. 22, p. 236. No ignoro, y con esto restrinjo mi observación, que uno de los grandes genios musicales del siglo xx, como lo es sin duda Olivier Messiaen, compartiría en todo y por todo la aseveración de Kant. Para Messiaen el canto de los pájaros es una suerte de epifanía de la divinidad. 
en el espíritu, "en cuanto sólo juega con sensaciones". Tal cual. Esto indica que según Kant la música sería un arte de lo agradable, que sólo place a los sentidos, es decir, a lo más exterior, pero no un arte bello en sentido estricto. Las notas pueden ser placenteras al sentido físico del oído, pero carecen de sustancia espiritual. Si se me permite una extrapolación anacrónica, diré que la posición de Kant ante la música en algo se parece a la que adopta Marcel Duchamp ante lo que él llama, con deliberado pulso crítico, una pintura "retiniana". Esta última sería aquella pintura conformista que complace al sentido de la vista pero que en esta misma medida carece de concepto, y no tiene por tanto ninguna repercusión intelectual digna de tomarse en consideración.

La única vez en toda la Crítica del juicio en que Kant cita un poema, además de que incurre en una cursilería involuntaria en tanto sensiblería de la época, muestra un espíritu cortesano, al transcribir los versos del rey Federico de Prusia, a quien además llama "el gran rey". He aquí la estrofa en cuestión:

Agótase nuestra vida sin murmullos ni quejas, abandonando el mundo después de haberlo colmado de beneficios.

Así, el sol, cuando ha terminado su carrera diurna, extiende aún por el cielo una luz dulce, y los últimos rayos que lanza en el aire son sus últimos suspiros por el bien del mundo. ${ }^{8}$

Un párrafo más adelante cita un verso de quien no da el autor, y que dice, en la misma tónica benéfica y reconfortante: "Manaba la luz del sol como la paz mana de la virtud". La evidente chabacanería de estos versos, empero, no le impide a Kant formular ahí mismo una noción que todavía hoy sigue pareciendo inquietante, o cuando menos, muy sugerente, la de las ideas estéticas, las cuales lograrían que en un concepto podamos pensar "muchas cosas inefables" y que no son susceptibles de representación. ${ }^{9}$

${ }^{8}$ Ibid., cap. 49, p. 284. En descargo de Kant, quisiera transcribir las siguientes afirmaciones de Sloterdijk: "[...] la Ilustración alemana posee no sólo representantes tales como Lessing y Kant, sino también a un Federico II de Prusia que hay que contar entre las cabezas de su siglo. Como príncipe fue hijo hecho y derecho de su época ilustrada, autor de un anti-Maquiavelo que repudia la técnica de dominio abiertamente cínica del anterior arte político [...] Con una coherencia formal y en parte también existencial aplicó a la Corona la idea de servicio al señalar al rey como 'primer servidor del Estado' [...]" (Peter Sloterdijk, Crítica de la razón cínica. Trad. de Miguel Ángel Vega. Madrid, Taurus, 1989, t. I., p. 121).

${ }^{9}$ Parece evidente que no sólo con su concepto del genio sino también con el de las "ideas estéticas", que servirían para articular lo inarticulable, esto es, para expresar lo "inefable", Kant prepara el terreno sobre el que cosecharán sus mejores frutos los pensadores y los poetas del romanticismo. 
En la historia de las ideas, la ubicación de Kant no deja de ser paradójica. Ilustrado a carta cabal, y enemigo por lo tanto de las rapsodias y de las efusiones del pensamiento que ya empezaban a manifestarse en su época, y por su insistencia en el sentimiento de lo bello y sus consideraciones acerca del genio, muchos consideran a Kant como uno de los padres del romanticismo. Transcribo la pertinente anotación de Isaiah Berlin, tomada de su libro Las raíces del romanticismo: "Kant odiaba el romanticismo. Detestaba toda forma de extravagancia, de fantasía, lo que él llamaba el Schwärmerei: cualquier tipo de exageración, misticismo, vaguedad, confusión. Sin embargo, se le considera con justicia uno de los padres del romanticismo, en lo que hay cierta ironía”. ${ }^{10}$

Puede asegurarse que si Kant hubiera visto a su hijo crecer, de seguro lo rechazaría como si se tratara de un monstruo. Lo que él propugnaba era un uso riguroso del entendimiento, único instrumento capaz de otorgarle autonomía al individuo y de sacar a la humanidad del estado lastimoso de minoría de edad en que la tenía sumergida la superchería y el fanatismo religioso. En su famoso artículo titulado "Respuesta a la pregunta: ¿Qué es la Ilustración?", Kant lo confirma:

Si ahora nos preguntáramos: ¿vivimos en una época ilustrada?, la respuesta sería: no, pero sí en una época de ilustración. Falta mucho todavía, tal como están las cosas, para que los hombres, tomados en conjunto, se hallen en situación o puedan estar dispuestos para servirse con seguridad y provecho de su propio entendimiento sin la guía de otro en materia de religión. Tenemos claras señales, sin embargo, de que se les ha abierto el campo para trabajar libremente y de que los obstáculos para la ilustración general, o para la salida del hombre de su culpable minoría de edad, son cada vez menos. Con esta perspectiva, esta época es la época de la ilustración, o el siglo de Federico. ${ }^{11}$

El sólo hecho de estimar que la Crítica del juicio abre las puertas al romanticismo que habría de venir, es ya considerar este libro desde un parámetro que rechazaba de manera explícita Kant, es leerlo desde una óptica distorsionada. El malentendido, sin embargo, es inevitable, a la vez que fructífero. La obra, una vez publicada, corre un camino y suscita experiencias y pensamientos de seguro no queridos por el autor, pero que pertenecen con todo derecho a la trascendencia histórica de una obra, a la historia de sus efectos,

${ }^{10}$ Isaiah Berlin, Las raíces del romanticismo. Madrid, Taurus, 2000, p. 99.

${ }^{11}$ I. Kant, En defensa de la Ilustración. Trad. de Javier Alcoriza y Antonio Lastra. Barcelona, Alba, 1999, pp. 69-70. 
como podría agregar Gadamer. Las Cartas sobre la educación estética del hombre de Schiller, son el mejor testimonio de esta recepción romántica provocada por el genio de Kant y de la que ya no podrá librarse en lo sucesivo. Kant, por ejemplo, no emplea nunca la palabra Einfühlung, traducida entre nosotros como "empatía" o "proyección sentimental", y que se convertirá en una de las divisas de la filosofía estética del romanticismo, pero sí la pronuncia uno de sus más cercanos discípulos, Herder. ${ }^{12}$

Desde mediados de los años cincuentas, filósofos y artistas muy diversos reciclan en provecho propio las aportaciones de Kant a la teoría de lo sublime. Un pintor abstracto estadounidense, Barnett Newman publica en 1948 una suerte de manifiesto que tendrá una amplia resonancia, "Es la hora de lo sublime" ("The Sublime is Now") en el que, aunque critica a Kant en razón de que éste en su teoría de la percepción trascendental habría entendido "que el fenómeno es más que un fenómeno", relanza la noción de lo sublime a título de ganzúa conceptual que le permite sacar del camino la estorbosa noción de lo bello. ${ }^{13}$ Inserto dentro de esta secuencia, y hasta radicalizando el gesto, Lyotard ha escrito que el concepto kantiano de lo sublime es la clave para entender el arte de nuestra época. Según Lyotard, el problema del arte contemporáneo es el de la "presentación de lo impresentable", que es el que se plantea la pintura abstracta de Rothko, de Pollock, de Jasper Johns y del mismo Barnett Newman. Lo impresentable o irrepresentable sería justamente lo sublime, de aquí la inesperada vigencia de Kant, mayor en este sentido que la de Hegel, quien en sus Lecciones sobre la estética habría arrinconado lo sublime al ámbito de lo que él llama el arte simbólico, o sea, el arte oriental, el arte primitivo o de lo monstruoso, caracterizado por la imperfección con la que concibe a la idea.

Me gustaría citar a Lyotard:

Llamaré moderno al arte que consagra su "pequeña técnica" [...] a presentar qué hay de impresentable. Hacer ver que hay algo que se puede concebir y que no se puede ver ni hacer ver: éste es el ámbito de la pintura moderna. ¿Pero cómo hacer ver que hay algo que no puede ser visto? El propio Kant nos dicta la dirección a seguir llamándolo lo in-

${ }^{12}$ El dato lo aporta Isaiah Berlin: "Einfühlen es de su invención, cien años antes de que Lipps, Dilthey o Croce la utilizaran" (vid. I. Berlin, Vico y Herder. Dos estudios en la historia de las ideas. Trad. de Carmen González del Trejo. Madrid, Cátedra, 2000, p. 223).

${ }^{13}$ Vid. Barnett Newman, "The Sublime is Now", en Charles Harrison y Paul Wood, eds., Art in Theory 1900-1990. An Anthology of Changing Ideas. Oxford, Blackwell, 1996. 
forme, la ausencia de forma, un índice posible de lo impresentable. Dice también de la abstracción vacía que experimenta la imaginación en busca de una presentación del infinito (otro impresentable) que esta abstracción es ella misma como una presentación del infinito, su presentación negativa. ${ }^{14}$

Tengo que decir a este respecto que Lyotard encarna de nuevo un malentendido más o menos feliz, pues en la Crítica del juicio Kant ilustra lo que él llama el sublime matemático y el sublime dinámico con fenómenos de la naturaleza, no con ejemplos artísticos, lo que ya tendría que dar materia de reflexión. Todo indica, en efecto, que Kant encuentra en la naturaleza el campo exclusivo de aplicación de lo sublime. Sólo una vez, de pasada, y más bien en el modo de una analogía, Kant relaciona el sentimiento de lo sublime con lo que podríamos considerar obras artísticas debidas al ingenio humano; lo hace cuando menciona las pirámides de Egipto y la catedral de San Pedro en el Vaticano, pero nada más. Fuera de ello, su concepción es a las claras restrictiva. Lo deja ya entrever en el capítulo 23, que es con el que comienza la "Analítica de lo sublime”. Ahí afirma: “...si como es justo, consideramos aquí primeramente sólo lo sublime en objetos de la naturaleza...” Lo correcto es, pues, ceñirse tan sólo a la naturaleza cuando se piensa en lo sublime. Para que no quede duda, pese a lo apretado de su formulación, agrega todavía entre paréntesis, de nuevo en un sentido restrictivo, y como en sotto voce: "(Lo sublime del arte se limita siempre a las condiciones de concordancia con la naturaleza)", con lo que habría que entender que sólo de modo derivado, y siempre que la pintura de un paisaje, por ejemplo, concuerde gracias a la eficacia mimética con la naturaleza que estaría ahí representada, podría hablarse de la presencia de lo sublime en el arte. ${ }^{15}$

${ }_{14}$ Jean-François Lyotard, La posmodernidad (explicada a los niños). Trad. de Enrique Lynch. Barcelona, Gedisa, 1996, p. 21. Tan está impresionado por la relevancia estética del pensamiento de Kant, y en particular por su atención al problema de lo sublime, que Lyotard ha escrito también unas Lessons on the Analytic of the Sublime. California, Universidad de Stanford, 1994. Este libro, todavía no traducido al español, es un detenido comentario de siete parágrafos de la Crítica del juicio de Kant, justo aquellos en los que aborda el asunto de lo sublime.

${ }^{15}$ I. Kant, Crítica del juicio, cap. 23, p. 237. No necesito mencionar, ya que es de todos conocido, que Kant tenía una franca predilección por lo bello en la naturaleza, y que sólo de modo derivado se ocupa de lo bello artístico. Este carácter secundario o derivativo del arte, retomado acaso de los estoicos, se patentiza en el enunciado suyo que acabo de citar. Hegel emblematiza en este sentido una verdadera ruptura, pues considera de modo primario a la belleza del espíritu, a la belleza producida por los hombres, al grado de asegurar que la belleza de una puesta de sol es sólo un fenómeno derivado de la belleza espiritual, en todo momento primaria, originaria. 
Observa Adorno en su Teoría estética: "Lo sublime que Kant reservó a la naturaleza se convirtió tras él en un constitutivo histórico del arte mismo". ${ }^{16}$ Esta reserva, conjetura Adorno acaso con razón, se debería a que el filósofo de Königsberg nunca se expuso a la experiencia estética del gran arte subjetivo que estaban empezando a producir por esos años los primeros románticos de su país. Como quiera que sea, las definiciones con las que apuntala su argumentación confirman que cuando Kant piensa en lo sublime lo piensa en relación con el espectáculo que ofrece la naturaleza. He aquí una de sus definiciones, casi tomada al azar: "Sublime es, pues, la naturaleza en aquellos de sus fenómenos cuya intuición lleva consigo la idea de su infinitud". ${ }^{17}$ Veamos de qué modo precisa lo sublime dinámico según esto en una naturaleza que no tendría sobre nosotros (sujetos sensibles) ningún poder: "La naturaleza, en el juicio estético, considerada como fuerza que no tiene sobre nosotros ningún poder, es dinámico-sublime" ${ }^{18}$ En el mismo tenor, establece un poco más adelante la esencia de lo sublime como aquello que "nos obliga a pensar subjetivamente la naturaleza misma en su totalidad, como exposición de algo suprasensible, sin poder realizar objetivamente esa exposición".${ }^{19}$ Como se ve, siempre se está refiriendo a la naturaleza.

Que Kant excluye en definitiva a lo sublime del arte lo podemos saber si acudimos a su Antropología en sentido pragmático, obra publicada ocho años después que la Crítica del juicio. En el capítulo 68 de su Antropología, Kant sostiene, me parece que de manera inequívoca: "la representación intelectual de lo sublime en la descripción o la plástica puede y tiene que ser siempre bella. En otro caso, tórnase el asombro espanto..." 20 Saturno devorando a uno de sus hijos, de Goya, no podría ser para Kant una obra de arte, puesto que no es bella, puesto que está más cerca del espanto que de la belleza. Lo sublime como tal, afirma Kant, no puede aparecer en el arte sino a condición de convertirse en otra cosa, es decir, a condición de dejar de ser sublime para aparecer bajo el manto reconfortante de la belleza. Sólo tenien-

16 Theodor W. Adorno, Teoría estética. Madrid, Taurus, 1992, p. 259. A propósito, para indicar hasta qué punto la Crítica del juicio ocupa un lugar central en las reflexiones de Adorno, me gustaría recordar que Robert Kaufman piensa que la Teoría estética reescribe la historia de la estética, pero a la manera de una espiral que avanzara progresivamente -y del modo más inesperado- de Hegel a Kant. Véase el ensayo de Robert Kaufman, "Red Kant, or The Persistence of the Third Critique in Adorno and Jameson", en Critical Inquiry. Verano, 2000, vol. 26, núm. 4, p. 710.

${ }^{17}$ Ibid., cap. 26, p. 244 . El subrayado es mío.

${ }^{18}$ Ibid., cap. 28, p. 248.

${ }^{19}$ Ibid., cap. 26, p. 253. Subrayados en el original.

${ }^{20}$ I. Kant, Antropología en sentido pragmático. Trad. de José Gaos. Madrid, Alianza, 1991, p. 173. 
do presente lo anterior se entiende la estricta lógica que ya desde las páginas iniciales de la Crítica del juicio nos dejaba saber que en el juicio estético de lo bello interviene el gusto, pero no así en el juicio referente a lo sublime. Lo sublime, de tal suerte, quedaba ya entonces excluido del gusto, lo cual se confirma en la obra que ahora comento. ${ }^{21}$

Las propuestas de Lyotard acerca de lo sublime, y con él las de toda una corriente de artistas y pensadores que se remiten de modo obligado a Kant para abordar este tema, incurren acaso sin saberlo en una extrapolación que no me parece trivial. Adulteran por una parte el pensamiento del filósofo, al aplicarlo a un ámbito de la cultura del que él lo había excluido de manera más o menos tajante, a la vez que lo inervan de una manera afortunada, al mostrar con tal amplificación la fecundidad (y sobre todo, la sorprendente actualidad) de sus consideraciones.

Kant parece más actual hoy en pleno remolino posmodernista que hace doscientos años, como ha sugerido Robert Kaufman en el texto antes señalado, y como lo demuestra la obsesión adorniana con Kant. De manera intersticial por no decir que insidiosa, muchos de los armónicos que se desprenden de los densos parágrafos de su Crítica del juicio, a la manera de clusters todavía no analizados, se incrustan como una lluvia de cristales sobre la piel de nuestra modernidad estética. El poderoso vacío kantiano como un medicamento homeopático continúa llenando los pavorosos huecos de nuestro pensamiento "actual", lo pongo debidamente entre comillas, como si la nada llamara a la nada y de ese modo lograra construir una Babel efímera, que a todos asombra y deja en el balbuceo. La bibliografía más reciente no me deja mentir. La tercera de las críticas continúa alzándose como un poderoso desafío intelectual al que de algún modo pensadores de muy distinto calibre están tratando de responder pese al lastre de las limitaciones tanto históricas como personales de quien la escribió. Señalo con estilo telegráfico algunas de estas limitaciones, todas más que evidentes.

Primero. La preferencia de Kant por lo bello natural en detrimento de lo bello artístico. Si Kant hubiera sido pintor, habría sido un paisajista como Jakob Phillip Hackert o como Caspar David Friedrich. Hegel fue quien primero reaccionó contra esta restricción intelectual, anteponiendo ante todo el poder del concepto.

Segundo. Su escasa cultura artística. No creo que tenga mucho sentido abundar en esto. Es lo que lo lleva a incluir la jardinería como una de las bellas artes.

${ }^{21}$ Reproduzco la esclarecedora posición de Kant, de una lógica impecable en un pensador de la época ilustrada: "La belleza es lo único que corresponde al gusto; lo sublime corresponde también al juicio estético, pero no al gusto" (I. Kant, Antropología en sentido pragmático, cap. 67, p. 171). 
Tercero. Las limitaciones históricas (y no sé si agregar, también, puritanas) de su juicio. Por poner un ejemplo, prefiere con mucho el dibujo al color. Acaso esto constituye un prejuicio de la época. Según Kant, los colores en la pintura estarían desvinculados de la idea, y ejercerían sólo un influjo por la vía del encanto, del hechizo sensorial, siempre reprobable desde el punto de vista estricto de un pietista como lo era Kant. Cito un pasaje que ilustra lo que quiero decir:

En la pintura, escultura, en todas las artes plásticas, en la arquitectura, en la traza de jardines, en cuanto son bellas artes, el dibujo es lo esencial; y en éste, la base de todas las disposiciones para el gusto la constituye, no lo que recrea en la sensación, sino solamente lo que, por su forma, place. Los colores que iluminan la traza pertenecen al encanto; ellos pueden ciertamente animar el objeto en sí para la sensación, pero no hacerlo digno de intuición y bello; más bien son, las más de las veces, muy limitados por lo que la forma bella exige, y aún allí donde se tolere el encanto, sólo por ella adquiere nobleza. ${ }^{22}$

Se diría que el color es como la carne, y que incita al pecado. Que propicia una suspensión o una anulación morbosa del juicio. Por eso la extraña frase que dimensiona centímetro a centímetro la castigada psicología kantiana: "y aún allí donde se tolere el encanto..." (subrayado mío), como si el encanto fuese por sí mismo algo negativo o perturbador que hubiese que evitar ante todo y a toda costa. Éste, en dado caso, según la prescripción kantiana, sólo por la forma podría adquirir carta de legitimación en sociedad.

\section{Recapitulación final acerca del a priori}

En alguna parte de la Fenomenología del espíritu, y como de pasada, Hegel anota que la conciencia es lo universal en sí. Lo universal en sí, esto es, aquello que se construye a partir de una cantidad infinita de negaciones por las que el intelecto consigue subyugar o reducir a polvo lo particular concreto. Una asociación de ideas me hace pensar en el a priori kantiano. Quizás no sea disparatado pensar el a priori de Kant como lo universal en sí, desvinculado de toda conciencia pero también anterior a ella, en un sentido riguroso y originario, en tanto que no puede dejar de constituirla. El a priori como una nada poderosísima puesta por la racionalidad al servicio de sus propios fines

${ }^{22}$ I. Kant, Crítica del juicio, cap. 14, p. 224. 
autónomos, como un gota de razón en el océano caótico de la empiria, a partir de la cual el océano puede por fin empezar a ser un océano, adquiriendo la forma que le hacía falta.

Sin el preconcepto, sin el a priori, sin lo universal en sí, pero un universal maquínico y autónomo que se impone previo a toda experiencia sobre la finitud del "ser finito pensante", expresión kantiana en la que me parece ver un primer atisbo de lo que habrá de ser el Dasein de Heidegger, el admirable edificio filosófico de Kant se viene abajo y pierde toda justificación. En la "Introducción" misma de la Crítica de la razón pura, Kant establece una distinción que constituye la esencia de su filosofía: "Pero, aunque todo nuestro conocimiento empiece con la experiencia, no por eso procede todo él de la experiencia”. Kant está convencido, y ésta es la aportación genial que él hace a la filosofía especulativa, que existen conocimientos a priori, los cuales, como la expresión lo dice, no provienen de la experiencia. Toda su formación empirista, debida sobre todo a su lectura de los filósofos ingleses, da un vuelco a partir de aquí, y se reconstituye sobre nuevas bases.

En la nota a pie de página que encontramos al inicio del capítulo dedicado a la "Estética trascendental" de la misma Crítica de la razón pura, al aludir a los esfuerzos heroicos de Baumgarten, Kant descartaba de plano la posibilidad de convertir a la estética en una ciencia. Vale la pena retomar de modo más completo su argumentación:

Los alemanes son los únicos que emplean hoy la palabra "estética" para designar lo que otros denominan crítica del gusto. Tal empleo se basa en una equivocada esperanza concebida por el destacado crítico Baumgarten. Esta esperanza consistía en reducir la consideración crítica de lo bello a principios racionales y en elevar al rango de ciencia las reglas de dicha consideración crítica. Pero este empeño es vano, ya que las mencionadas reglas o criterios son, de acuerdo con sus fuentes principales, meramente empíricas y, consiguientemente, jamás pueden servir para establecer determinadas leyes a priori por las que debiera regirse nuestro juicio de gusto. ${ }^{23}$

Me parece evidente que si Kant hubiera permanecido toda su vida fiel a este lapidario punto de vista, nunca hubiera escrito la tercera de sus críticas. Darse a la tarea de redactar la Crítica del juicio, implica reconsiderar algo que había sido excluido y modificar una antigua concepción, que con el paso de los años se ha revelado como equívoca y hasta cierto punto como insoste-

${ }^{23}$ I. Kant, Crítica de la razón pura, pp. 66-67. 
nible. ${ }^{24}$ Gracias a esta rectificación que mucho tiene de genial y todavía más de inesperado es que existe la Crítica del juicio. Lo que haría falta ver es qué se ha conservado de esa antigua nota en el nuevo libro, si es que algo ha quedado de ella, y cuál es el descubrimiento que le permite a Kant pisar un terreno al que la convicción de otro tiempo le prohibía hacerlo.

Por principio, hay que dejar en claro que el Kant de la Crítica de la razón pura y el de la Crítica del juicio coinciden en un aspecto fundamental. Una ciencia de lo bello como tal es una ciencia imposible. Lo dice en la nota que acabo de transcribir, y lo refrenda nueve años después en el capítulo 44 de la Crítica del juicio, al que ya aludí antes en la nota 4 de este mismo trabajo: "No hay ni una ciencia de lo bello, sino una crítica, ni una ciencia bella, sino sólo arte bello..." Ésta es la razón sistemática por la cual Kant no podría haber escrito una estética: pensaba que la estética como ciencia era un despropósito, una quimera inalcanzable. En este punto, el Kant de la tercera crítica sigue subscribiendo puntualmente lo que decía el Kant de la primera en esa nota a la que aludí con anterioridad, y que podría resumirse en este argumento toral: no nos es dado encontrar ni establecer las "leyes a priori" que pudieran regir el juicio de gusto. Es la aleatoriedad inescapable del juicio de gusto lo que impide constituir una estética.

Entonces, ¿qué significa escribir una Crítica del juicio, y qué es lo que hizo posible que se escribiera? Si Kant no se ha movido un solo centímetro de su antigua posición, en la medida en que sigue pensando que no es dable encontrar "leyes a priori" que rijan el juicio de gusto, sí en cambio ha descubierto que existe un principio a priori que rige el funcionamiento específico del juicio y hasta que lo hace posible. No una legalidad apriorística como tal, en toda la extensión del término, pero sí en cambio un principio a priori colocado en la base del juicio, y que elimina con ello su supuesta condición aleatoria. De la aparición sorprendente de este matiz depende que se escriba la tercera de las críticas.

Es la emergencia inesperada del a priori lo que le descubre a Kant un campo antes inexplorado. Este campo es el juicio, siempre que se le entienda no como el resultado de una actividad empírica de la inteligencia, sino como un ejercicio sintético de las facultades de conocimiento del sujeto que estaría fundado apriorísticamente. Ya en la "Introducción" a la Crítica del juicio, Kant se da el lujo de declarar: "Tan lejos como se extienda la aplicación de conceptos a priori se extiende el uso de nuestra facultad de conocer según

${ }^{24}$ Tengo para mí que la mencionada nota era un verdadero candado epistemológico, que el mismo Kant tuvo que hacer saltar en pedazos para poder continuar con su tarea especulativa. 
principios, y con él la filosofía”. ${ }^{25}$ ¿Cómo ha ampliado Kant la filosofía de su época? Proponiendo que el juicio también tiene un principio de este tipo. Por eso afirma Kant: "hay motivo para suponer, por analogía, que [el juicio] encierra en sí igualmente, si no una legislación propia, al menos su propio principio, uno subjetivo, a priori, desde luego, para buscar leyes..."26

Según Kant, el concepto a priori del juicio reflexionante es el de finalidad. Gracias al principio de la finalidad de la naturaleza, ésta puede ser representada "como si un entendimiento encerrase la base de la unidad de lo diverso de sus leyes empíricas". (Subrayado mío). Surge así el principio heurístico de teleología que permite que el sujeto pueda conferirle unidad al caos fenoménico de la naturaleza. En lo que respecta al juicio estético, Kant encuentra esta misma finalidad encarnada en el conocimiento de la forma del objeto. Cuando la imaginación (como facultad de la exposición) concuerda de modo espontáneo y como sin proponérselo con el entendimiento (como facultad de los conceptos) por medio de una representación dada, de aquí nace un sentimiento de placer que constituye la esencia del juicio estético. Este sentimiento placentero debe ser considerado como el resultado y el objeto final del juicio reflexionante, como su producto más nítido. Es la finalidad interna propia del juicio, no importa que se trate como sucede siempre en el juicio estético, de una finalidad sin fin, ajena a todo sentido de provecho y de utilidad. Que esta finalidad preceda al juicio, que sea aún más un requisito apriorístico del mismo, es lo que le permite a Kant conjurar el fantasma contingente de lo empírico y colocarse en una posición especulativa, quiero decir, filosóficamente pertinente. Al liberarse de lo particular concreto, al trascender el espinoso campo de lo empírico, el juicio infinito kantiano instituye no como punto de llegada sino como origen más originario lo que Hegel llamaba lo universal en sí, una nada del raciocinio que permite dar orden a todo raciocinio.

${ }^{25}$ I. Kant, "Introducción", en Crítica del juicio, p. 191.

${ }^{26}$ Ibid., p. 193. Como Venus de la espuma de la mar, así brota el juicio reflexionante del océano insondable del a priori kantiano. El pasaje que acabo de citar tiene una importancia estratégica, y bien podría ser la piedra miliar de todo el edificio. 